\title{
The Challenge to Diagnose ADHD in Adults
}

\author{
Maria Tereza V.Gouvea, Hospital Municipal Dr A. R. Saboya, Sao Paulo
}

\section{Background}

ADHD is relatively easy to diagnose in children. However, in adults the different clinical presentation, may lead to not consider this diagnose or its comorbidity, which do not allow correct treatment

\section{introduction}

ADHD in adults is often disregarded in psychiatric consultation. Individuals with mild symptoms of ADHD in childhood, which didn't have strong negative impacts in learning abilities and also those who had mild hyperactivity symptoms, and no behavior problems, are not usually identified in childhood, as often is the case in girls. Meanwhile, as the complex tasks of adult life, demands higher levels on executive functions, the difficulties related to ADHD may start to appear, only in adulthood. Patients struggling to deal with the symptoms of ADHD, without knowledge of the condition, have complaints that are not clearly linked to a history of ADHD, They can not be easily recognized without a careful interview and assesment with specific scales to establish this diagnosis should be more often applied.

\section{Material and Methods}

Based on clinical observation, we present four cases, of adults that initially had not been diagnosed with ADHD, neither with comorbidity with ADHD, and we made this diagnosis.

The adapted ASRS was applied.

\section{Case 1 Previous Diagnose Anxiety Disorder}

36 year old male patient, that had been previously diagnosed with anxiety disorder. He had received for many years treatment with antidepressants and anxyolitics with no amelioration, In the interview he described what he called as anxiety, an intense mental activity, impatience, restlesness and impulsivity, being constantly worried about things that he thinks he should have done. He was said to be an agitaded child.

Although he had good learning abilities, he had a perception of not progressing professionally accordingly.

He scored positive in the screening for ADHD and decided upon knowledge of his condition not to take medicaments, but to try a behavior therapy approach.

\section{Case 2 Previous Diagnose Recurrent Depressive Disorder}

31 year old male patient, feeling discouraged, presenting himself with low self stem, low confidence, frustration by poor achievements in professional life, despite excellent academic background, due to inability to fit in structured employs, to finish projects, to focus in priorities $\mathrm{He}$ had received different antidepressants, with poor response, and showed good improvement of symptoms with the introduction of metylphenidate.

\section{Case 3 Substance Use Disorder}

A 41 years old male patient, that besides having severe problems due to drug addiction, as we could observe in some others with comorbidity of ADHD and SUD, is able to maintain a relatively stable social life and a skilled employ. During subsequently office visits, he voiced complaints that made us investigate for symptoms of ADHD. He expressed attraction for being involved in dangerous situations, lack of concentration, mental hyperactivity, does different things at the same time, has a feeling of boredom without use of cocaine. When he was only treated for SUD, he had frequent relapses and the prescription of metylphenidate has provided a better outcome

\section{Case 4 Previous Diagnosis Bipolar Disorder}

A 32 years old female patient had made a suicide attempt, impulsively, without depression symptoms. She had been diagnosed and treated previously, for bipolar disorder, with no amelioration in irritability, insomnia, acceleration of thoughts. She fulfilled clinical criteria for ADHD. The symptoms of hyperactivity, lack of concentration, were present in childhood, impaired her at school years, but were not recognized as being due to ADHD. This patient we were not able to do the follow up. She was addressed for ADHD treatment in another center.

\section{Conclusion}

Complaints of discouragement associated with professional underachievement, procrastination, difficulty in finishing tasks, lack of concentration, impatience, loosing objects frequently, history of hyperactivity in childhood, anxiety, agitation, relentless, impulsivity,

should be explored in adults in order to better diagnose ADHD and allow its treatment. 